\title{
Tratamento de queloide retroauricular: Revisão dos casos tratados no serviço de otorrinolaringologia do HC/UFPR
}

\section{Treatment of retroauricular keloids: Revision of cases treated at the ENT service of HC/UFPR}

\author{
Bettina Carvalho', Annelyse Cristine Ballin², Renata Vecentin Becker ${ }^{3}$, Talita Beithum Ribeiro ${ }^{3}$, Juliana Benthien Cavichiolo', \\ Carlos Roberto Ballin ${ }^{4}$ Marcos Mocellin ${ }^{5}$.
}

1) Graduaçao em Medicina pela UFPR. Médica Residente do Serviço de Otorrinolaringologia do HC/UFPR.

2) ORL pela ABORL. Médica Otorrinolaringologista.

3) Acadêmica de Medicina da UFPR.

4) Mestre em Cirurgia pela UFPR. Responsável pelo Setor de Cirurgia Crânio Maxilo Facial do Serviço Otorrinolarinolaringologia do HC/UFPR.

5) Doutorado em Otorrinolaringologia pela Escola Paulista de Medicina, Brasil (1986). Professor Titular da Universidade Federal do Paraná, Brasil Chefe do Serviço de Otorrinolaringologia do HC/UFPR.

Instituição: Hospital de Clinicas da UFPR. Curitiba / PR - Brasil.

Endereço para correspondências: Bettina Carvalho - Rua General Carneiro, 181 - Centro, Curitiba / PR - Brasil - CEP: 80060-900 - E-mail: bettinacarvalho@ yahoo.com.br Artigo recebido em 14 de julho de 2011. Artigo aprovado em 19 de setembro de 2011.

\section{RESUMO}

Introdução: Queloides são tumores benignos resultantes de cicatrização anômala da pele, sendo que existem diversos procedimentos disponíveis para o seu tratamento.

Objetivo: O objetivo deste trabalho é avaliar os resultados de pacientes submetidos a tratamento de queloides retroauriculares após cirurgias otorrinológicas em nosso serviço.

Método: Estudo retrospectivo e prospectivo, através de análise de prontuários, dos pacientes submetidos a tratamento de queloides retroauriculares em nosso serviço.

Resultados: Nove pacientes foram avaliados, sendo que 6 foram submetidos a ressecção e betaterapia adjuvante, 2 foram submetidos a ressecção mais aplicação de corticoide local, 1 foi submetido apenas a ressecção sem terapia adjuvante. Não houve recidiva nos casos tratados com betaterapia no pós-operatório precoce, 1 paciente apresentou recidiva mesmo com corticoterapia e betaterapia tardia.

Discussão: diversas técnicas tem sido utilizadas para tratamento dos queloides retroauriculares, sendo que a betaterapia é considerada a com melhores resultados, seguida pela utilização de corticoides intralesionais.

Conclusão: o tratamento dos queloides retroauriculares continua um desafio. Enquanto novas técnicas não são desenvolvidas, a ressecção seguida de betaterapia precoce ainda é a melhor opção.

Palavras-chave: queloide, terapêutica, orelha.

\section{SUMMARY}

Introduction: Keloids are benign tumors arising from abnormal healing of the skin, and there are several procedures available for their treatment.

Objective: The objective of this study was to evaluate the outcomes of patients undergoing treatment of keloids after ear, nose, and throat (ENT) surgeries at our service center. Method: We conducted thorough, retrospective and prospective analysis of records of patients undergoing treatment of retroauricular keloids at our center.

Results: Nine patients were evaluated, and 6 underwent resection and adjuvant beta-therapy, 2 underwent resection with local application of corticosteroids, and only 1 underwent resection without adjuvant therapy. There was no recurrence of keloids in patients that were treated with beta-therapy in the early postoperative period. One patient had relapsed despite corticosteroid administration and late beta-therapy.

Discussion: Several techniques have been used for the treatment of retroauricular keloids, and beta-therapy is thought to yield the best results, followed by the use of intralesional corticosteroids.

Conclusion: Treatment of retroauricular keloids remains a challenge. While new techniques are being developed, resection followed by early beta-therapy is still the best treatment option.

Keywords: keloid, treatment outcome, ear. 


\section{INTRODUÇAO}

Queloides são tumores benignos fibrosos (1) que surgem como reações anormais de cicatrização da pele. Os queloides se desenvolvem a partir do tecido conectivo como resposta a trauma como inflamação, queimaduras, "piercings" ou cirurgias emáreas de alta tensão no corpo, em indivíduos predispostos $(1,2)$. Podem ocorrer em qualquer idade e não tem preferência por sexo, mas tendema ocorrer mais frequentemente em indivíduos entre 10 e 30 anos. Há uma predominância maior em pacientes de pele escura e áreas de orelha, ombros e tórax (3).

O queloide se apresenta como uma cicatriz espessa e lisa (2) que se extende alem dos limites da ferida original e pode ter um relevo elevado na pele, levando a deformidade local (3). Geralmente começam a crescer dentro do 1o ano apos o trauma e raramente regridem (2). Os principais sintomas associados aos queloides são formigamento, ardência e prurido, levando a alterações funcionais e desconforto (1).

A nível celular a característica do queloide é a síntese descontrolada e depósito excessivo de colágeno e glicoproteína (2). Já foi demonstrado que os queloides são compostos por fibroblastos policlonais, intrinsecamente normais, mas que respondem anormalmente a sinais extracelulares, resultando em uma produção excessiva e degradação diminuída do tecido fibrótico cicatricial (1).

Seu diagnóstico diferencial é feito com as cicatrizes hipertróficas, que são cicatrizes de margens confinadas à ferida original e geralmente mostram regressão espontânea (2). Esta distinção é muito importante, pois essas entidades merecem tratamentos diferenciados.

A abordagem retroauricular e muito usada em otorrinolaringologia, tanto em cirurgias de orelha média (timpanoplastia, timpanomastoidetomia), como de orelha externa (otoplastia), funcionais ou meramente estéticas, sendo portanto, local potencial de origem de queloides devido a ser uma área de alta tensão da pele.

O objetivo deste trabalho é avaliar a epidemiologia dos pacientes com queloide (idade, sexo, cor da pele, procedimento que originou o queloide) e os resultados de pacientes submetidos a tratamento de queloides retroauriculares após cirurgias otorrinológicas em nosso serviço.

\section{MÉTODO}

Este trabalho foi aprovado pelo Comitê de Ética em Pesquisa em Seres Humanos do HC/UFPR.
Foi realizada revisão de prontuários de pacientes submetidos a tratamento de queloides retroauriculares no serviço de Otorrinolaringologia do HC/UFPR no periodo de 2006 a 2009 e avaliados prospectivamente os pacientes submetidos a ressecção do queloide em 2010, através de um protocolo e fotografias pré e pós-operatórias.

Foram avaliados o tipo de cirurgia que resultou no queloide, realização de cirurgia para ressecção do queloide, tratamentos adjuvantes pós-operatórios (betaterapia, aplicação intralesional de corticoide), número de recidivas e reoperações, características da recidiva (presença de hiperemia, telangiectasias, coloração, tamanho da cicatriz), sintomas associados (dor, prurido), satisfação do paciente quanto ao resultado.

\section{RESULTADOS}

Foram avaliados nove pacientes ( 13 orelhas), com idades entre 9 e 27 anos, a distribuição conforme o sexo se deu da seguinte forma: 6 homens e 3 mulheres.

Quatro pacientes evoluíram com queloides após cirurgias de otoplastia, 2 após timpanoplastia, 1 após mastoidectomia, 1 após cirurgia para correção de malformação congênita (sinus Pré-auricular) e 1 após cirurgia realizada em outro serviço, não podendo ser descrita.

Seis pacientes foram submetidos a sessões de betaterapia no Hospital Erasto Gaertner, entre estes 2 haviam iniciado infiltração com corticoide, e foram submetidos a betaterapia tardiamente.

Dois pacientes receberam apenas infiltração com corticoide local (betametasona 3mg, Celestone Soluspam ${ }^{\circledR}$ ), e 01 paciente não foi submetido a terapia adjuvante pelo queloide ser muito pequeno.

Apos o uso dos tratamentos adjuvantes, mantemos o uso de pomada de triancinolona $1 \mathrm{mg}\left(\right.$ Oncilom $\left.^{\circledR}\right) \mathrm{com}$ massagem local, por cerca de 2 meses.

\section{Os resultados estão dispostos na Tabela 1 e Figuras} $1,2,3$.

A técnica cirúrgica é ilustrada na Figura 4. A cirurgia é realizada da seguinte forma: anestesia local atraves da infiltração de Lidocaína 2\% + Adrenalina 1:50.000, marcação das margens da cicatriz queloide com violeta de genciana, incisão em forma de fuso, descolamento por planos da cicatriz, confecção de um retalho por deslizamento das margens, hemostasia, sutura simples da ferida operatória, curativo. 
Tabela I. Resultados.

\begin{tabular}{|c|c|c|c|}
\hline Paciente & Girurgia prévia & Tratamento & Recidiva \\
\hline । & Bilateralapós otoplastia & Ressecção + betaterapia + pomada*** & Semrecidiva \\
\hline 2 & Bilateralapóstimpanoplastia & Ressecção + betaterapia + pomada*** & $\begin{array}{l}\text { Semrecidiva } O E \\
\text { discretahipertrofia } \bigcirc D\end{array}$ \\
\hline 3 & Bilateralapós otoplastia & Ressecção unilateral(lado maior) + betaterapia & Semrecidiva \\
\hline 4 & Bilateralapós otoplastia & Ressecção + CTC***** + pomada** & Semrecidiva \\
\hline 5 & Unilateralapós mastoidectomia & Ressecção + CTC ****** + betaterapia* + pomada*** & Recidiva \\
\hline 6 & Unilateralapóstimpanoplastia & Ressecção + CTC****** & Semrecidiva \\
\hline 7 & Unilateralapós otoplastia & $\begin{array}{l}\text { Ressecção + reconfecção das suturas mestras } \\
\text { de mustarde + betaterapia }\end{array}$ & Semrecidiva \\
\hline 8 & $\begin{array}{l}\text { Unilateralapós correção de } \\
\text { malformação congênita }\end{array}$ & Ressecção apenas & Semrecidiva \\
\hline 9 & Unilateral & Ressecção + betaterapia + CTC***** & Semrecidiva \\
\hline
\end{tabular}

* betaterapia iniciada tardiamente ( 2 meses após)

** pomada com corticoide triancinolona $1 \mathrm{mg}\left(\right.$ Oncilom $\left.{ }^{\circledR}\right)$

*** CTC = corticoterapia com injeção intralesional de betametasona 3mg (Celestone Soluspan $\left.{ }^{\circledR}\right)$

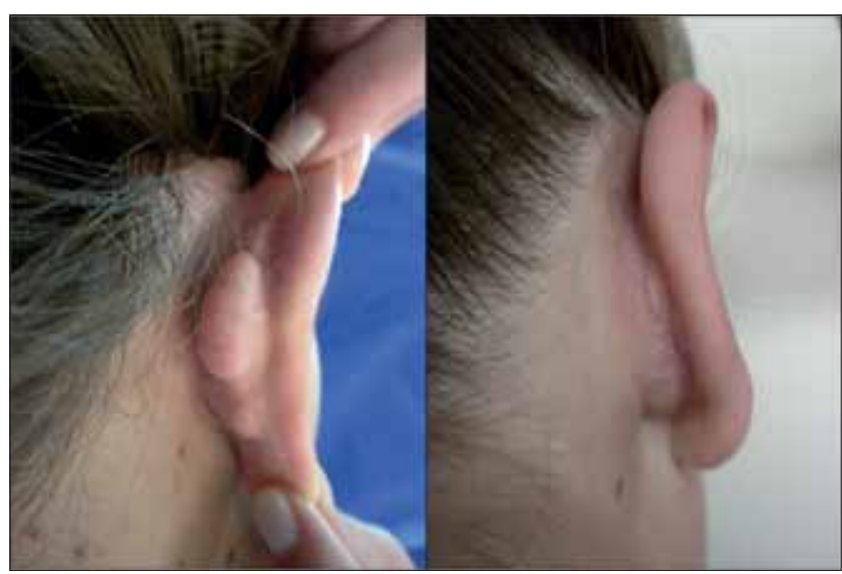

Figura 1. Caso 3: Pré-operatório (à esquerda) e pós-operatório de 3 meses (à direita).

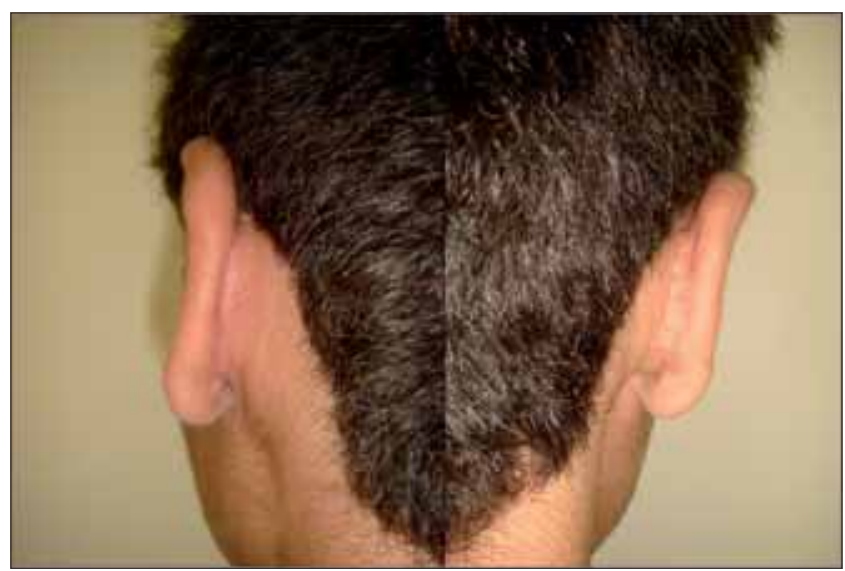

Figura 3. Caso 1: Pós-operatório de 1 ano e 1 mês.

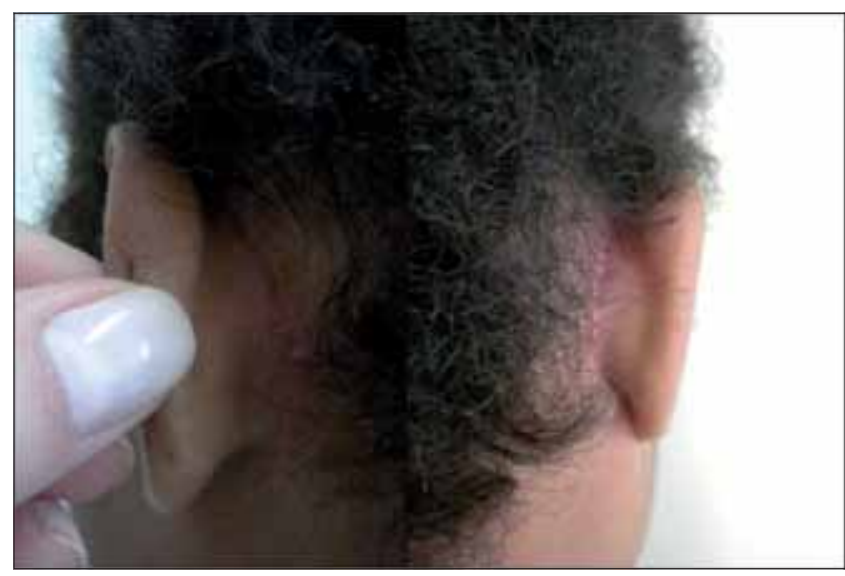

Figura 2. Caso 2: Pós-operatório de 3meses.

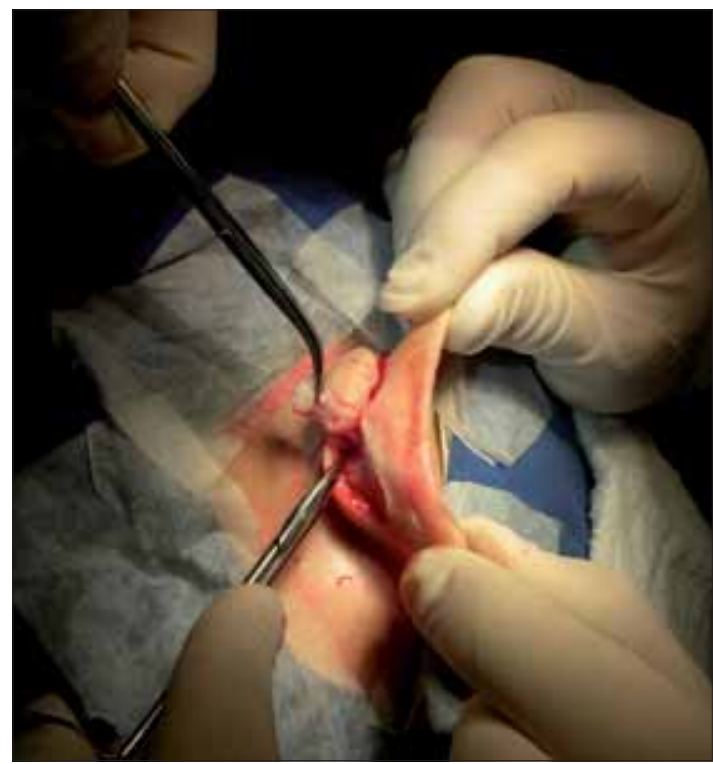

Figura 4. Técnica cirúrgica - ressecção do queloide retroauricular em fuso com retalho por deslizamento. 
A betaterapia é realizada com placas de Estrôncio $\left.{ }^{90} \mathrm{Sr}\right)$. A fonte da betaterapia é uma placa de $2 \mathrm{~cm}$, acondicionada em recipiente conforme a Figura 5. As intruções para uso são de que a placa deve permanecer fora do recipiente apenas durante o periodo de tratamento, sendo que o técnico deve manter a maior distância possível durante a aplicação, e a fonte de radiação ainda possui uma placa de acrílico que serve de barreira para a radiação. O paciente é orientado a fazer lavagem do local a ser irradiado e secar bem a pele, não é utilizado nenhum produto ou aplicação no local durante a irradiação. O tempo e a quantidade de irradiação em Grays varia de acordo com cada paciente, dependendo da extensão da ferida.

\section{DISCUSSÃO}

Os queloides são um problema que afeta a qualidade de vida dos pacientes, não só pela questão estética, mas principalmente pelos sintomas associados (prurido, dor, sensação de queimação e intolerância a alguns tecidos), (4) fazendo-se necessário o emprego de um tratamento específico eficaz.

A maior característica e dificuldade no tratamento dos queloides é a sua recorrência apos exérese cirúrgica. Para resolver este problema, diversas modalidades de tratamentos adjuvantes vem sendo desenvolvidas.

Entre as diversas opções de tratamento usadas para prevenir a recorrência de queloides estão: pressão contínua após cirurgia, corticoides intralesionais, laser de dióxido de carbono, aplicação de gel de silicone, administração de ácido retinoico, cobertura com gel de silicone (1), criocirurgia, uso de agentes quimioterápicos (5-fluorouracil), interferon intralesional, radioterapia, entre outros (5). No entanto, não há evidencia sólida de que estes métodos são efetivos na prevenção da recidiva, sendo a radiotera pia o único tratamento que mostrou ser efetivo na prevenção da formação de queloides (1).

É importante lembrar que o queloide formado não é radiossensivel, porem os fibroblastos de cicatrizes recentes o são, sendo portanto necessário aplicar-se a radiação no tecido conjuntivo no pós-operatório imediato, ou seja, na fase de proliferação e diferenciação dessas células e não no tecido fibrótico formado (4).

As técnicas de radioterapia empregadas incluem: radioterapia externa com raios $\mathrm{X}$ superficiais, braquiterapia com estrôncio Sr-90 ou feixes de elétrons, braquiterapia de dose baixa (1) , braquiterapia de alta dose $(6,7)$, radioterapia pré associada a pós-operatória (8).

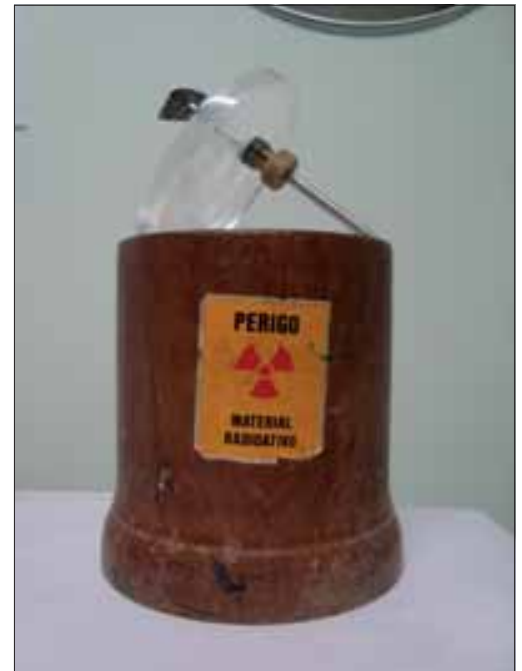

Figura 5. Recipiente da placa de Estrôncio (Betaterapia).

O mecanismo pelo qual a radioterapia age não é bem esclarecido, porém acredita-se que a radiação destrua os fibroblastos, que são substituídos por células provenientes da corrente sanguínea, provindas de outros tecidos (1).

Áreas com excessiva curvatura da pele podem levar a uma maior dificuldade de entrega da radiação pósoperatória. E a manipulação e compressão para aplainamento das áreas recém-operadas pode não ser bem tolerada, ou levar a danos para a área recentemente suturada. HuHn et al (9) demonstram uma técnica de radioterapia pós-operatória visando manter a posição anatômica da orelha, minimizando a manipulação da ferida operatória enquanto maximiza a dose de radiação à área alvo. O meato auditivo externo é protegido com uma bola de algodão e um escudo de chumbo. A concha é preenchida com uma material moldável (Suertuff bolus ${ }^{\circledR}$ ), criando uma estrutura plana e uniforme ao nível da hélice e trágus. Outro material (Superflab ${ }^{\circledR}$ ) é utilizado para preencher o espaço retroauricular, formando uma superfície continua com a hélice, antihélice e lóbulo. Estima-se que este arranjo manteria as áreas alvo dentro dos 95\% da dose limite do raio de elétrons.

Em nosso serviço encaminhamos os pacientes submetidos a ressecção de queloides ao serviço de radioterapia do Hospital Erasto Gaertner (hospital oncológico) para serem submetidos a betaterapia pós-operatória.

A betaterapia é realizada através do uso de placas de Sr 90 (Estrôncio 90 - radioisótopo), em doses de cerca de 30 Gy (Gray = unidade de dose absorvida) (4).

Segundo Hochman et al (10) a associação de cirurgia com betaterapia é a melhor opção de tratamento, realizan- 
do-se a betaterapia 24 a 48 horas após a excisão, com o intuito de atenuar a fibroplasia.

Em nosso serviço conseguimos bons resultados com o uso da betaterapia quando realizada precocemente (um paciente iniciou a betaterapia no mesmo dia da cirurgia), em 4 pacientes. Um paciente apenas conseguiu realizar a betaterapia tardiamente ( 2 meses de pós-operatório), apresentando recidiva, mesmo com uso de infiltração com corticoide precoce.

A radioterapia percutânea pós-operatória tem poucos efeitos adversos como o escurecimento da pele no local tratado (11), mas não tem mostrado efeitos adversos graves, como surgimento de tumores, mesmo em acompanhamentos a longo prazo $(4,11)$.

Dentre as outras variedades de técnicas adjuvantes no tratamento dos queloides, há a injeção intralesional de corticoide (geralmente triancinolona) para prevenção de queloides ou tratamento de queloides já existentes na ferida operatória. A administração pode ser intraoperatoria e pós-operatória, e tem mostrado redução da taxa de recidiva dos queloides (11). Segundo Hochman et al (10), reduz a recidiva para menos de $50 \%$.

BERMUElLer et al (12) conseguiram bons resultados cosméticos e alto grau de satisfação dos pacientes com ressecção e confecção de retalho de pele completo para queloides retroauriculares e fechamento por primeira intenção para queloides em lóbulo de orelha associada a infiltração de corticoide.

Em nosso serviço, para prevenção de queloides pós-operatórios, os corticoides intralesionais são utilizados no pós-operatório imediato, $1 \mathrm{~mL}$ por aplicação, em aplicações espaçadas por 2 semanas, nos primeiros 2 meses de pós-operatório. Utilizamos a betametasona $3 \mathrm{mg}$ (Celestone soluspam $\left.{ }^{\circledR}\right)$. Apesar de ainda preferirmos a betaterapia, é uma alternativa para tratamento quando aquela não é possível ou não é desejada pelo paciente. Quatro pacientes realizaram aplicações de corticoide apos ressecção cirúrgica, combons resultados.

Os principais efeitos colaterais locais são atrofia, despigmentação e telangiectasias da cicatriz tratada e, apesar de pouco frequentes, pode haver reações adversas sistêmicas ao uso de corticoides, mesmo que em aplicação intralesional. Liu e Yемсна (13) relataram um caso de Síndrome de Cushing iatrogênica após o uso de injeções intralesionais de triancinoloma para o tratamento de múltiplos queloides. Os autores sugerem, portanto, considerar o tamanho e numero de queloides envolvidos no tratamento usando corticoides intralesionais. Levando-se em consideração a recorrência e agressividade da doença, pode haver a necessidade de grandes volumes e frequência de injeções e a potencialidade de induzir efeitos sistêmicos, devendo os pacientes terem a função adrenal monitorada antes de sinais da síndrome aparecerem.

Por outro lado, o risco de recorrências, independentemente do tipo de tratamento adjuvante utilizado, depende de fatores locais relacionados à ferida operatória e fatores gerais relacionados ao paciente. Uma recorrência maior de queloides é vista em locais onde a ferida é suturada com tensão, ou existe infecção pós-operatória e deiscência da sutura. Pacientes com história familiar positiva e história prévia de queloides recidivantes com falhas terapêuticas prévias tem maior risco de recorrência $(10,11)$.

Em nosso estudo, um paciente não foi submetido a terapia adjuvante, porem seu queloide era pequeno, não apresentou recidiva e mantem-se satisfeito com o resultado obtido.

O pico de incidência da recidiva ocorre aproximadamente até o $13^{\circ}$ mês, por isso o acompanhamento dos pacientes com queloides deve ser longo (10). Em nosso serviço os pacientes mantem acompanhamento por pelo menos 1 ano apos o término do tratamento.

Apesar de os queloides serem mais frequentes em pessoas de etnia negra, em nosso serviço encontramos todos os pacientes classificados como de etnia branca. Segundo Hochman et al (10), as taxas de recidiva não mostram relação com a etnia, idade ou tamanho da lesão.

A nível bioquímico, foi observado em estudos um aumento da expressão da citocina TGF- $\beta$ e de seus receptores em fibroblastos de tecidos queloidianos. A família da TGF- $\beta$ tem como efeito fisiológico promover crescimento e proliferação de células, inibir apoptose, induzir síntese de ciclooxigenase-2 que promove crescimento de invasão do tumor ao produzir angiogênese ou secreção de metaloproteinase-2 (MM-2). GAO et al (14) mostraram um aumento da expressão do gene da GDF-9 (membro da família da TGF- $\beta$ ) nos fibroblastos da região periférica do tecido queloidiano, área responsável pelo crescimento e invasão de tecidos normais. Sugerem então que os fibroblastos que produzem GDF-9 sejam um dos responsáveis pela invasão tecidual periférica.

UlRich et al mostraram que a formação de tecido de granulação e cicatrização da ferida dependem de um balanço adequado entre a síntese e degradação de matriz extracelular, sendo que uma síntese excessiva ou degradação deficiente ou ambas podem levar a formação de cicatrizes patológicas, como os queloides. As enzimas envolvidas neste processo são as metaloproteinases (Matrix Metaloproteinases - MMP), enzimas que degradam com- 
ponentes da matriz extracelular, que sao inibidas pelas Tissue inbibitors of metalloproteinases(TIMP). No futuro, os autores consideram que tratamentos não-cirurgicos que diminuam a expressão de TIMP's e aumentem a de MMP podem ser terapias para o tratamento de cicatrizes patológicas, como os queloides (15).

Enquanto este futuro não chega, o tratamento dos queloides, inclusive os retroauriculares, continua um desafio.

\section{CONCLUSÃO}

O tratamento dos queloides retroauriculares apresenta muitas dificuldades, seja com relação ao tratamento do queloide em si, seja em relação a localização.

Em nosso serviço, conseguimos bons resultados com a exérese cirúrgica associada a betaterapia iniciada logo após a cirurgia.

Espera-se que, com o desenvolvimento de outras terapias não cirúrgicas, o tratamento dos queloides retroauriculares e de outros queloides também seja facilitado.

\section{REFERENCIAS BIBLIOGRAFICAS}

1. Viani GA, Stefano EJ, Afonso SL, De Fendi LI . Posoperative strontium-90 brachytherapy in the prevention of keloids: results and prognostic factors. Int $J$ Radiation Oncology Biol Phys. 2009, 73(5):1510-1516.

2. Froelich K, Staudenmaier R, Leinsasse N, Hagen R. Therapy of auricular keloids: review of different treatmente modalities and proposal for a therapeutic algorithm. Eur Arch Otorhinolaryngol. 2007, 264:1497-1508.

3. Correspondence and Comunication. Use of colchicine to prevent recurrence of ear keloids. A new approach. Journal of Plastic, Recontructive and Aesthetic Surgery. 2010, 63: e650-e652.

4. Oliveira Jr B, Lastoria JC, Pereira HR, Silveira LVA, Oliveira LP, Stolf HO. Estudo comparativo entre o tratamento radioterápico com elétrons e betaterapia, após cirurgia de quelóides. Surgical \& Cosmetic Dermatology. 2009, 1(2):5357.

5. Durani P, Bayat A. Levels of evidence for the treatment of keloid disease. Jouranl of Plastic, Reconstructive and Aesthetic Surgery. 2008, 61:4-17.

6. Garg MK, Weiss P, Sharma AK, Gorla GR, Jaggernauth W, Yaparpalvi R, Delrowe J, Beitler JJ. Adjuvant high dose brachytherapy (Ir-192) in the managemente of keloids which have recurred after surgical excision and external radiation. Radiotherapy and Oncology. 2004, 73:233-236.

7. Veen RE, Kal HB. Postoperative high-dose-rate brachytherapy in prevention of keloids. Int. J. Radiation Oncology Biol Phys. 2007, 69(4):1205-1208.

8. Stahl S, Barnea Y, Weiss J, Zaretski A, Leshem D, Miller E, Shafir R, Ben-yosef R, GurE. Treatment of earlobe keloids by extralesional excision combined with preoperative and postoperative "sandwich" radiotherapy. Plastic and Reconstructive Surgery. January 2010,125(1).

9. Huhn JL, Johnson EL, St Clair W. Adjuvant radiation of bilateral postauricular keloids: an illustration of technique. Medical dosimetry 2007, 32(4):278-280.

10. Hochman B, Locali RF, Oliveira LQR, Ferreira LM. Disfunções cicatriciais hiperproliferaticas: Quelóide. Estima. 2004, 4(4):33-39.

11. Ragoowansi R, Cornes PGS, Glees JP, Powell BW, Moss LH. Ear-lobe keloids: treatment by a protocol of surgical excision and immediate postoperative adjuvant radiotherapy. British Journal of Plastic Surgery. 2001, 54 : 504-508.

12. Bermueller C, Rettinger G, Keck T. Auricular keloids: treatment and results. Eur Arch Otorhinolaryngol. 2010, 267:575-580.

13. Liu MF, Yencha M. Cushing's syndrome secondary to intralesional steroid injections of multiple keloids. Otolaryngology-Head and Neck Surgery. 2006, 135:960961.

14. Gao Z, Wu X, Song N, Zhang L, Liu W. Differential expression of growth differentiation factor-9 in keloids. Burns. 2010.

15. Ulrich D, Ulrich F, Unglaub F, Piatkowski A, Pallua N. Matrix metalloproteinases and tissue inhibitors of metalloproteinases in patients with different types of scars and keloids. Journal of Plastic, Reconstructive \& Aesthetic Surgery. 2010, 63:1015-1021. 